\title{
Economic burden of antibiotic resistance in China: a national level estimate for inpatients
}

\author{
Xuemei Zhen ${ }^{1,2}$, Cecilia Stålsby Lundborg ${ }^{3}$, Xueshan Sun ${ }^{1}$, Nina Zhu ${ }^{4}$, Shuyan Gu ${ }^{1,5}$ and Hengjin Dong ${ }^{1,6^{*}}$ (D)
}

\begin{abstract}
Background: Antibiotic resistance (AR) threats public health in China. National-level estimation of economic burden of AR is lacking. We aimed to quantify the economic costs of AR in inpatients in China.

Methods: We performed a multicentre and retrospective cohort study including 15,990 patient episodes at four tertiary hospitals in China from 2013 to 2015 to assess the impact of AR on hospital mortality, length of stay, and costs. We estimated the societal economic burden of AR using findings from the cohort study and secondary data from national surveillance hubs and statistical reports.

Results: Patients with multi-drug resistant (MDR) infection or colonisation caused by Staphylococcus aureus, Enterococcus faecalis, Enterococcus faecium, Escherichia coli, Klebsiella pneumonia, Pseudomonas aeruginosa, and Acinetobacter baumannii experienced higher individual patient cost (\$3391, 95\% uncertainty interval (UI) \$3188-3594), longer hospital stay (5.48 days, 95\% UI 5.10-5.87 days), and higher in-hospital mortality rates (1.50\%, 95\% UI 1.29-1.70\%). In China, $27.45 \%$ of bacterial infection or colonisation that occurred in inpatients were resistant, of which $15.77 \%$ were MDR. A societal economic burden attributed to AR was estimated to be $\$ 77$ billion in 2017, which is equivalent to $0.37 \%$ of China's yearly gross domestic product, with $\$ 57$ billion associated with MDR.

Conclusions: This is the first study to estimate national-level economic burden of AR in China. AR places a significant burden on patient health and healthcare systems. Estimation of economic costs of resistant infection or colonisation is the essential step towards building an economic case for global and national actions to combat AMR.
\end{abstract}

Keywords: Antibiotic resistance, Multi-drug resistance, Economic burden, Inpatient, China

\section{Background}

Antibiotic resistance (AR) threatens the effective prevention and treatment of an ever-increasing range of infections caused by bacteria and places one of the greatest threats on global health systems [1]. This problem is particularly severe in China, associated with overuse and misuse of antibiotics in human and animals [2, 3]. China was the second largest consumer of antibiotics in 2010 in the globe. In primary care facilities, $52.9 \%$ of outpatient prescriptions and $77.5 \%$ of inpatient prescriptions

\footnotetext{
*Correspondence: donghj@zju.edu.cn

${ }^{1}$ Center for Health Policy Studies, School of Public Health, Zhejiang University School of Medicine, Hangzhou, China

Full list of author information is available at the end of the article
}

contained antibiotics, of which, only $39.4 \%$ and $24.6 \%$ were considered appropriate respectively [4]. Among BRICS countries (Brazil, Russia, India, China, and South Africa), more than $57.0 \%$ of the increase in antibiotic consumption in hospital occurred in China between 2000 and 2010 [5].

Driven by the inadequate consumption of antibiotics, China has the world's most rapid growth of AR [6]. The proportion of methicillin-resistant Staphylococcus aureus (MRSA), third-generation cephalosporin resistant Escherichia coli (3GCREC), third generation cephalosporin resistant Klebsiella pneumonia (3GCRKP), carbapenem resistant $K$. pneumonia (CRKP), carbapenem resistant Pseudomonas aeruginosa (CRPA), carbapenem resistant Acinetobacter baumannii (CRAB) in original author(s) and the source, provide a link to the Creative Commons licence, and indicate if changes were made. The images or other third party material in this article are included in the article's Creative Commons licence, unless indicated otherwise in a credit line to the material. If material is not included in the article's Creative Commons licence and your intended use is not permitted by statutory regulation or exceeds the permitted use, you will need to obtain permission directly from the copyright holder. To view a copy of this licence, visit http://creativecommons.org/licenses/by/4.0/. The Creative Commons Public Domain Dedication waiver (http://creativeco mmons.org/publicdomain/zero/1.0/) applies to the data made available in this article, unless otherwise stated in a credit line to the data. 
China in 2017 were 32.2\%, 54.2\%, 33.0\%, 9.0\%, 20.7\%, and $56.1 \%$, respectively, which were higher than those in Europe (16.9\%, 14.9\%, 31.2\%, 7.2\%, 17.4\%, and 33.4\%) [7-9].

AR is associated with prolonged hospital stay, higher medical costs and increased hospital mortality [10]. In the European Union and European Economic Area countries, it was estimated that AR attributed to $€ 1.1-$ 1.5 billion economic loss 33,000 deaths each year [11, 12]. In the United States (US), AR led to approximately $\$ 55$ billion excessive healthcare costs and subsequent societal costs and 35,000 deaths each year [7, 13].

However, in China, similar analysis to estimate the burden of AR on national level is lacking. Despite the recent recognition of the public health threat posed by $\mathrm{AR}$ and the development of a national action plan for antimicrobial resistance (AMR) [14], limited information as to its economic burden hinders the country's progress in addressing AR [15]. Robust economic assessments of AR are urgently needed if the top-level political commitment is to be enforced [16]. In this study, we aimed to estimate the national level economic burden of AR in China for inpatients. First, we estimated the number of inpatients with AR in China; then, we estimated the societal economic burden for inpatients in China due to AR.

\section{Methods}

\section{Study setting}

We collected data in four tertiary hospitals in China, three in Zhejiang Province (Site 1, Site 3, and Site 4), and one in Shandong Province (Site 2). Site 1 and Site 2 are general provincial hospitals, Site 3 is a general county hospital, and Site 4 is a provincial hospital providing healthcare integrated with traditional Chinese medicine (Table 1). We selected these hospitals as study sites due to their relatively complete hospital information system, which can make reliable data collection possible.

\section{Patient enrollment}

This was a retrospective study. During the study period between January 2013 and December 2015, we extracted data from the electronic medical records (EMR) of the study sites for patients who had bacterial infection or colonisation confirmed by any clinical specimens (e.g. blood, urine, stool, cervical, or urethral sources) [17]. Only $60 \%$ of inpatients with positive samples from Site 1 and $40 \%$ cases from Site 2 were randomly selected due to the large inpatient population, and 100\% cases from Site 3 and Site 4 were selected. We only included the first sample if there were multiple samples from the same isolate during the study period in order to avoid duplication. If a patient was re-admitted in the sampled hospitals, we recorded as multiple cases.

We extracted patient information, including patient demographics (age, sex, and health insurance), comorbidities (disease diagnosis, and Charlson Comorbidity Index (CCI)), hospital events (admitting service, surgical service, and date of hospital and intensive care unit (ICU) admission or discharge), and clinical outcomes (death or alive during the hospitalization when discharged) from first page of medical record system, microbiological data from microbiology laboratory, and costs for treatments from financial system. We matched different data from different system using patient identification, and patients with missing above information were excluded.

\section{Pathogen selection and case categorisation}

In this study, we included patients with infection or colonisation caused by $S$. aureus, E. faecalis, E. faecium, E. coli, K. pneumonia, $P$. aeruginosa, and $A$. baumannii. We classified the infection or colonisation cases into susceptible episodes and resistant episodes (including resistant and intermediate isolates) based on the susceptibility test results for the patient specimens. The susceptible

Table 1 The characteristics of the study settings during 2013 and 2015

\begin{tabular}{|c|c|c|c|c|}
\hline Characteristics & Site 1 & Site 2 & Site 3 & Site 4 \\
\hline Province & Zhejiang & Shandong & Zhejiang & Zhejiang \\
\hline Number of beds & 3200 & 3500 & 1727 & 2100 \\
\hline Number of discharged inpatients yearly & 170,000 & 160,000 & 80,000 & 50,000 \\
\hline $\begin{array}{l}\text { Number of patients enrolled (\% of inpatients with total positive bacterial } \\
\text { infection or colonisation) }\end{array}$ & $4541(60)$ & $2535(40)$ & 4993(100) & $3921(100)$ \\
\hline Number of patients with resistant infection or colonisation (SDR) & $1112(20.37)$ & $720(13.19)$ & $1789(32.77)$ & 1838(33.67) \\
\hline Number of patients with resistant infection or colonisation (MDR) & $2282(30.97)$ & $1427(19.37)$ & $1851(25.12)$ & $1808(24.54)$ \\
\hline Number of patients with susceptible infection or colonisation & $1147(36.26)$ & $388(12.27)$ & $1353(42.78)$ & $275(8.69)$ \\
\hline
\end{tabular}

SDR single-drug resistant, MDR multi-drug resistant 
cases were in control group. The AR cases as case group were further categorised into either single-drug resistant (SDR) or multi-drug resistant (MDR). We defined SDR cases if patients were resistant to at least one antibiotic drug in one or two antibiotic categories, and MDR cases were defined as patients resistant to one antibiotic drug in three or more antibiotic categories. The interpretation of susceptibility test results was based on the Clinical and Laboratory Standards Institute (CLSI) definitions [8, 18].

\section{Statistical analysis}

We conducted propensity score matching (PSM) to eliminate selection bias by balancing the potential confounding variables between susceptible and resistant infection or colonisation cases [19]. Patient demographics, comorbidities (cancer, diabetes or not), disease characteristics (CCI, number of diagnosis), and treatment (admission to ICU, surgery) were as independent variables. We employed 1:1 nearest-neighbor matching with a 0.05 caliper value, and the propensity score was balanced when there were no differences in baseline characteristics between the two groups. The outcome measure are individual patients' hospital costs, length of hospital stay, and in-hospital mortality rate using matched pairs. We performed 1000 iterations of Monte Carlo simulations to calculate the $95 \%$ uncertainty interval (UI) for each outcome measure with normal distribution. Time discounting and age weighting were not considered in this study [20].

The definition, value and data source of the input equation parameters are presented in Fig. 1. We parameterised the equation using data collected from the study sites before mentioned and data reported in published literature. We used the data from the latest year available for parameters from secondary data sources. If national level estimates are not available, we used the data collected from the study sites to represent the national population. The rates of MRSA, 3GCREC, FQREC, 3GCRKP, CRKP, CRPA, and CRAB among inpatients with positive bacterial culture from four sampled hospitals were $48.87 \%, 55.73 \%, 58.65 \%, 32.51 \%, 10.29 \%, 31.75 \%$, and $56.54 \%$, respectively, which are similar to the country's average level reported by China Antimicrobial Resistance Surveillance System (34.42\%, 57.17\%, 52.82\%, 35.05\%, $8.03 \%, 22.62 \%$, and $58.05 \%$ ) [8]. The average total hospital cost, length of hospital stay, and in-hospital mortality rate in Zhejiang province and Shandong province in 2017 were $\$ 3252$ and $\$ 2604,9.8$ days and 8.6 days, $0.3 \%$ and $0.4 \%$, which were approximate to the national average (\$2557, 9.3 day, and 0.4\%) [21].

\section{Estimation of AR in inpatients in China}

We estimated the number of inpatients with AR using primary data collected from the study cohort and secondary source data. We calculated total number of inpatients with AR infection or colonisation in China by multiplying total number of inpatients with prescribed antibiotics in China and proportion of AR infection or colonisation among inpatients in China. Except for total population in China and hospital admission rate, we did not consider age- and gender-specific attack rates due to sample size and data availability.

First, we extracted total population in China in 2017 [22] and hospital admission rate in 2013 [21] with age and gender distribution from United Nations (UN) and China Health Statistical Yearbook, respectively. Second,

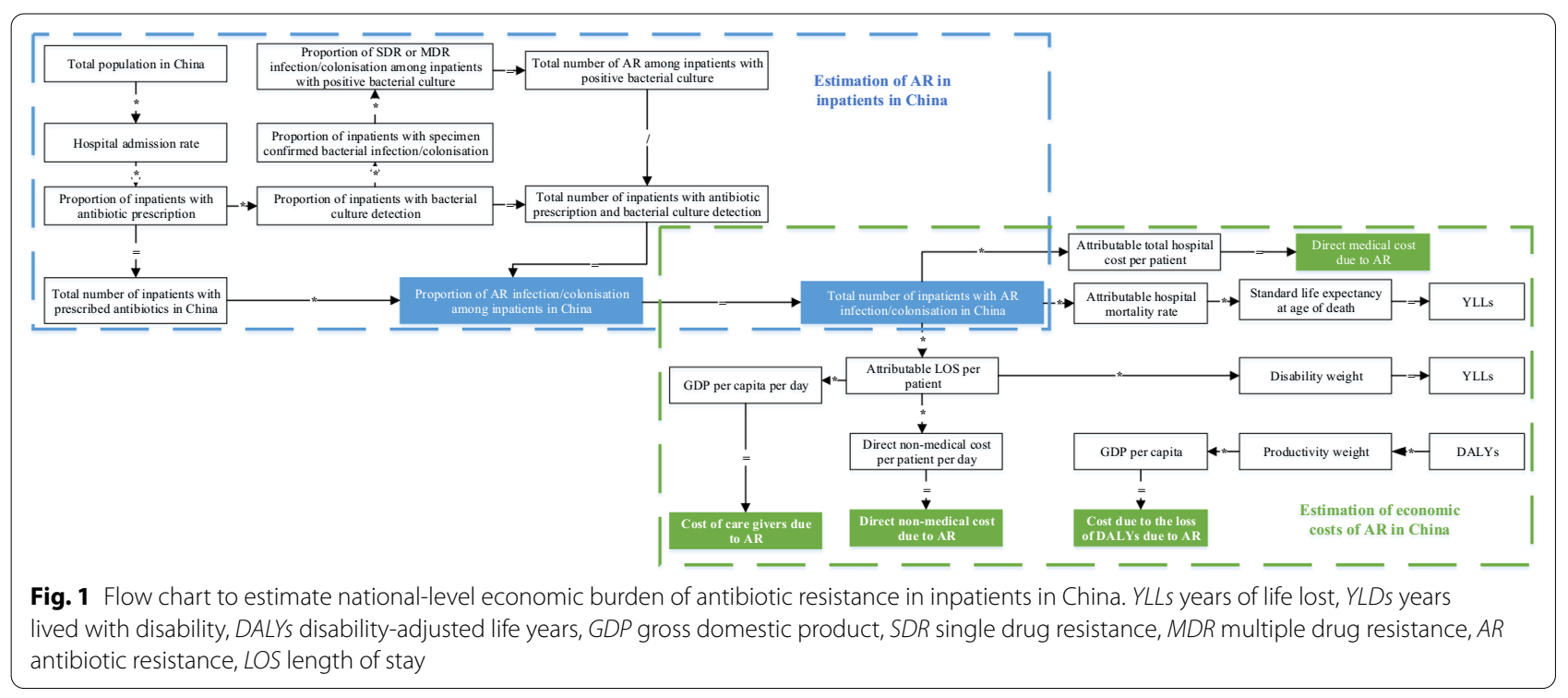


we collected proportion of inpatients with antibiotic prescription, proportion of inpatients with bacterial culture detection, and proportion of inpatients with specimen-confirmed bacterial infection or colonisation from National Nosocomial Infection Survey 2014. Third, proportion of SDR and MDR infection or colonisation among inpatients with positive bacterial culture was calculated using EMR from sampled hospitals between 2013 and 2015. Last, we calculated proportion of AR infection/ colonisation among inpatients in China and total number of inpatients with AR infection/colonisation in China (Fig. 1, Additional file 1: Figure S1, Table S1, Table S2).

\section{Estimation of economic costs of AR in China}

We calculated the economic costs of inpatients with AR by summing direct medical, direct non-medical, and indirect costs. All hospital costs were presented in 2015 US dollar values using the purchasing power parities and the 2015 consumer price index of China [23, 24]. Medical costs included out-of-pocket payment (by patients themselves) and payment covered by health insurers for medication and materials, diagnostic tests, treatment procedures, and other cost during a patient's hospital stay. We calculated direct medical cost due to AR by multiplying attributable total hospital costs per patient and total number of inpatients with AR in China.

Attributable direct non-medical cost included costs for accommodation, meals, transport during the patient's hospital stay. We used data from a previous survey for direct non-medical costs per day paid by individual hospitalised patients [25]. Then, we calculated direct nonmedical cost due to AR by multiplying attributable direct non-medical stay per patient per day, length of hospital stay per patient and total number of inpatients with AR in China.

Attributable indirect costs included costs of care givers and productivity loss measured in disability-adjusted life years (DALYs) due to AR. Variables using to calculate DALYs included standard life expectancy at age of death from the UN [22], productivity weight and disability weight from Global Burden of Disease 2013, and GDP per capita in 2017 from China Statistical Yearbook [26]. Cost of care givers due to AR was calculated by multiplying GDP per capita per day, attributable length of hospital stay per patient and total number of inpatients with AR in China. Cost due to the loss of DALYs due to AR was calculated by multiplying DALYs, productivity weight and GDP per capita. Due to the small sample size, DALYs calculations used average mortality instead of age-specific mortality and DALYs is the sum of years of life lost (YLLs) (multiplying total number of inpatients with AR in China, attributable hospital mortality rate, and standard life expectancy at age of death) and years lived with disability (YLDs) (multiplying total number of inpatients with AR in China, attributable length of hospital stay per patient, and disability weight) (Fig. 1).

\section{Ethics approval}

The institutional review board of Zhejiang University School of Public Health reviewed and approved this study. All patient data has been anonymised prior to analysis.

\section{Results}

\section{Demographic characteristics of the study cohort}

We collected 3163, 5459, and 7368 inpatients with susceptible, SDR and MDR infection or colonisation from four sampled hospitals, The demographic characteristics of the patients included in the study cohort are presented in Tables 2 and 3. Older age, higher proportion of insurance coverage, lower number of diagnostics, lower CCI, higher ICU admission rate, and higher surgical admission rate were associated with the risk of developing SDRinfection or colonisation. Older age, being female, higher proportion of insurance coverage, higher number of diagnostics, higher CCI, higher ICU admission rate were associated with the risk of developing MDR-infection or colonisation.

Differences in comorbidities were also observed between groups of SDR-, MDR- and susceptible infection or colonisation. We performed PSM for 3135 pairs of SDR- and susceptible infection or colonisation, and 3048 pairs of MDR- and susceptible infection or colonisation. After PSM, the characteristics mentioned above appeared to be insignificant (Tables 2, 3).

\section{Number of inpatients with AR in China}

The secondary data used to estimate the national-level proportion of AR in China was presented in Additional file 1, including total population with gender and age distribution (Additional file 1: Figure S1), hospital admission rates aggregated by gender and age group (Additional file 1: Table S1), and proportion of bacterial infection or colonisation (Additional file 1: Table S2). We estimated $12,098,752$ inpatients (27.45\% of all inpatients) had AR infection or colonisation nationwide, including 5,113,276 (11.68\%) with SDR and 6,985,476 (15.77\%) with MDR among all among inpatients using the secondary data before mentioned and the proportion of susceptible, SDR and MDR infection and colonisation in our study cohort (Fig. 2).

\section{Economic costs associated with AR}

Compared with patients with susceptible infection or colonisation, the mean differences in total hospital cost, length of hospital stay, and in-hospital mortality rate 
Table 2 Baseline characteristics of inpatients with SDR and susceptible infection or colonisation before and after PSM

\begin{tabular}{|c|c|c|c|c|c|c|}
\hline \multirow[t]{2}{*}{ Baseline characteristics } & \multicolumn{3}{|l|}{ Before PSM } & \multicolumn{3}{|l|}{ After PSM } \\
\hline & Susceptible & SDR & P-value & Susceptible & SDR & $P$ value \\
\hline Number of inpatients, $\mathrm{n}$ & 3163 & 5459 & & 3135 & 3135 & \\
\hline Age in year, median (Min-Max) & $68(0-100)$ & $73(0-100)$ & $<0.000$ & $68(0-100)$ & $69(0-100)$ & 0.994 \\
\hline Sex male, $\mathrm{n}(\%)$ & $1904(60.2)$ & $3324(60.9)$ & 0.525 & $1881(60.0)$ & $1873(59.7)$ & 0.837 \\
\hline Insurance, n (\%) & $2529(80.0)$ & $4727(86.6)$ & $<0.000$ & $2525(80.5)$ & $2526(80.6)$ & 0.975 \\
\hline Number of diagnosis, median (Min-Max) & $6(1-36)$ & $6(1-30)$ & 0.0002 & $6(1-36)$ & $6(1-30)$ & 0.925 \\
\hline CCl, median (Min-Max) & $5(1-37)$ & $5(1-33)$ & $<0.000$ & $5(1-37)$ & $5(1-33)$ & 0.815 \\
\hline Admission to ICU, n (\%) & $335(10.6)$ & $415(7.6)$ & $<0.000$ & $329(10.5)$ & $321(10.2)$ & 0.740 \\
\hline Surgery, n (\%) & $923(29.2)$ & $1161(21.3)$ & $<0.000$ & $904(28.8)$ & $920(29.4)$ & 0.656 \\
\hline Myocardial infraction, n (\%) & $92(2.9)$ & $125(2.3)$ & 0.077 & $90(2.9)$ & $85(2.7)$ & 0.701 \\
\hline Congestive heart failure, n (\%) & $620(19.6)$ & $959(17.6)$ & 0.019 & $603(19.2)$ & $611(19.5)$ & 0.798 \\
\hline Peripheral vascular disease, n (\%) & $45(1.4)$ & $48(0.9)$ & 0.019 & $41(1.3)$ & $39(1.2)$ & 0.822 \\
\hline Cerebrovascular diseases, n (\%) & $1428(45.2)$ & $2665(48.8)$ & 0.001 & $1423(45.4)$ & $1369(43.7)$ & 0.170 \\
\hline Dementia, n (\%) & $45(1.4)$ & $254(4.7)$ & $<0.000$ & $45(1.4)$ & $40(1.3)$ & 0.585 \\
\hline Chronic pulmonary disease, n (\%) & $969(30.6)$ & $1388(25.4)$ & $<0.000$ & $960(30.6)$ & $969(30.9)$ & 0.805 \\
\hline Connective tissue disease, n (\%) & $80(2.5)$ & $132(2.4)$ & 0.748 & $80(2.6)$ & $75(2.4)$ & 0.684 \\
\hline Mild liver disease, $\mathrm{n}(\%)$ & $109(3.5)$ & $194(3.6)$ & 0.794 & $109(3.5)$ & $122(3.9)$ & 0.383 \\
\hline Peptic ulcer disease, $n$ (\%) & $69(2.2)$ & $141(2.6)$ & 0.244 & $69(2.2)$ & $65(2.1)$ & 0.727 \\
\hline Diabetes mellitus, n (\%) & $795(25.1)$ & $1546(28.3)$ & 0.001 & $795(25.4)$ & $813(25.9)$ & 0.603 \\
\hline Diabetes mellitus with chronic complication, n (\%) & $112(3.5)$ & $242(4.4)$ & 0.044 & $112(3.6)$ & $104(3.3)$ & 0.580 \\
\hline Moderate to severe chronic kidney disease, n (\%) & $212(6.7)$ & $447(8.2)$ & 0.012 & $211(6.7)$ & $217(6.9)$ & 0.764 \\
\hline Hemiplegia, n (\%) & $20(0.6)$ & $54(1.0)$ & 0.083 & $20(0.6)$ & $17(0.5)$ & 0.621 \\
\hline Solid tumor without metastases, n (\%) & $223(7.1)$ & $425(7.8)$ & 0.212 & $223(7.1)$ & $23397.4)$ & 0.627 \\
\hline Leukemia, n (\%) & $37(1.2)$ & $66(1.2)$ & 0.872 & $37(1.2)$ & $38(1.2)$ & 0.908 \\
\hline Malignant lymphoma, n (\%) & $24(0.8)$ & $50(0.9)$ & 0.446 & $24(0.8)$ & $27(0.9)$ & 0.673 \\
\hline Severe liver disease, n (\%) & $34(1.1)$ & $81(1.5)$ & 0.111 & $34(1.1)$ & $36(1.2)$ & 0.810 \\
\hline Metastatic tumor, n (\%) & $137(4.3)$ & $304(5.6)$ & 0.012 & $137(4.4)$ & $133(4.2)$ & 0.803 \\
\hline
\end{tabular}

SDR single-drug resistant, CCI Chalson comorbidity index, ICU intensive care unit, PSM propensity score matching

were $\$ 1144$ (95\% UI \$965-\$1322), 4.09 days (95\% UI 3.70-4.47 days), $0.78 \%$ (95\% UI 0.59-0.96\%) in patients with SDR infection or colonisation, and \$3391 (95\% UI \$3188-\$3594), 5.48 days (95\% UI 5.10-5.87 days), $1.50 \%$ (95\% UI $1.29-1.70 \%)$ in patients with MDR infection or colonisation (Table 4, 5). In addition, GDP per capita in 2017 and direct non-medical cost per person was $\$ 15,011$ [21] and \$88 (95\% UI \$85-\$91), respectively. We set productivity weight as $0.15,0.75,0.80,0.1$ for aged 0-14 years, $15-44$ years, $45-59$ years, above 60 years, respectively [27]. Disability weight was derived from Global Burden of Disease 2013 with a value of 0.133 (95\% UI 0.332-0.190) [22]. Standard life expectancy at age of death was presented in Table S3 [22].

We estimated a total societal economic cost attributed to AR in inpatients in China of $\$ 77$ billion (95\% UI $\$ 67$ billion-\$87 billion), including $\$ 35$ billion (95\% UI $\$ 32$ billion- $\$ 38$ billion) of direct cost and $\$ 42$ billion (95\% UI $\$ 35$ billion- $\$ 49$ billion) of indirect cost. The attributable total economic cost is equivalent to $0.37 \%$ of China's
GDP in 2017, among which, $\$ 20$ billion (95\% UI \$16 billion-\$24 billion) was caused by SDR infection or colonisation, and \$ 57 billion (95\% UI \$ 51 billion-\$ 63 billion) by MDR infection or colonisation (Table 6 ).

\section{Discussion}

Quantifying the economic costs of resistant infection or colonisation is the essential step towards building an economic case for global and national actions to combat AMR. To our knowledge, this study is the first study to estimate economic burden of AR in China at national level.

We estimated a percentage of $27.45 \%$ inpatients with resistant infection or colonisation, which is similar to the figure reported in previous studies conducted on regional level in China [6]. We predicted a total number of 7.0 million inpatients with MDR infection or colonisation in China, which is significantly higher than the number of 2.8 million in the US [13] and of 0.7 million in Europe [11]. In addition, The attributable economic 
Table 3 Baseline characteristics of inpatients with MDR and susceptible infection or colonisation before and after PSM

\begin{tabular}{|c|c|c|c|c|c|c|}
\hline \multirow[t]{2}{*}{ Baseline characteristics } & \multicolumn{3}{|l|}{ Before PSM } & \multicolumn{3}{|l|}{ After PSM } \\
\hline & Susceptible & MDR & P-value & Susceptible & MDR & P-value \\
\hline Number of inpatients, $\mathrm{n}$ & 3163 & 7368 & & 3048 & 3048 & \\
\hline Age in year, median (Min-Max) & $68(0-100)$ & $72(0-102)$ & $<0.000$ & $68(0-100)$ & $70(1-100)$ & 0.955 \\
\hline Sex male, n (\%) & $1904(60.2)$ & $4168(56.6)$ & 0.001 & $1805(59.2)$ & $1815(59.6)$ & 0.794 \\
\hline Insurance, n (\%) & $2529(80.0)$ & $6122(83.1)$ & $<0.000$ & $2469(81.0)$ & $2424(79.5)$ & 0.148 \\
\hline Number of diagnosis, median (Min-Max) & $6(1-36)$ & $6(1-37)$ & $<0.000$ & $6(1-36)$ & $6(1-37)$ & 0.945 \\
\hline CCl, median (Min-Max) & $5(1-37)$ & $5(1-39)$ & $<0.000$ & $5(1-37)$ & $5(1-28)$ & 0.626 \\
\hline Admission to ICU, n (\%) & $335(10.6)$ & $1133(15.4)$ & $<0.000$ & $333(10.9)$ & $356(11.7)$ & 0.352 \\
\hline Surgery, n (\%) & $923(29.2)$ & $2239(30.4)$ & 0.215 & $899(29.5)$ & $965(31.7)$ & 0.067 \\
\hline Myocardial infraction, n (\%) & $92(2.9)$ & $197(2.7)$ & 0.499 & $87(2.9)$ & $95(3.1)$ & 0.547 \\
\hline Congestive heart failure, n (\%) & $620(19.6)$ & $1193(16.2)$ & $<0.000$ & $577(18.9)$ & $569(18.7)$ & 0.793 \\
\hline Peripheral vascular disease, n (\%) & $45(1.4)$ & $93(1.3)$ & 0.507 & $43(1.4)$ & $48(1.6)$ & 0.597 \\
\hline Cerebrovascular diseases, n (\%) & $1428(45.2)$ & $3444(46.7)$ & 0.132 & $1392(45.7)$ & $1410(46.3)$ & 0.644 \\
\hline Dementia, n (\%) & $45(1.4)$ & $277(3.8)$ & $<0.000$ & $45(1.5)$ & $45(1.5)$ & 1.000 \\
\hline Chronic pulmonary disease, n (\%) & $969(30.6)$ & $1451(19.7)$ & $<0.000$ & $867(28.4)$ & $894(29.3)$ & 0.445 \\
\hline Connective tissue disease, n (\%) & $80(2.5)$ & $189(2.6)$ & 0.915 & $80(2.6)$ & $96(3.2)$ & 0.221 \\
\hline Mild liver disease, n (\%) & $109(3.5)$ & $321(4.4)$ & 0.030 & 109 (3.6) & $104(3.4)$ & 0.727 \\
\hline Peptic ulcer disease, n (\%) & $69(2.2)$ & $199(2.7)$ & 0.121 & $68(2.2)$ & $71(2.3)$ & 0.797 \\
\hline Diabetes mellitus, n (\%) & $795(25.1)$ & $2137(29.0)$ & $<0.000$ & $791(26.0)$ & $775(25.4)$ & 0.639 \\
\hline Diabetes mellitus with chronic complication, n (\%) & $112(3.5)$ & $293(4.0)$ & 0.286 & $112(3.7)$ & $108(3.5)$ & 0.784 \\
\hline Moderate to severe chronic kidney disease, n (\%) & $212(6.7)$ & $713(9.68)$ & $<0.000$ & $212(7.0)$ & $194(6.4)$ & 0.355 \\
\hline Hemiplegia, n (\%) & $20(0.6)$ & $101(1.4)$ & 0.001 & $20(0.7)$ & $17(0.6)$ & 0.621 \\
\hline Solid tumor without metastases, n (\%) & $223(7.1)$ & $729(9.9)$ & $<0.000$ & $223(7.3)$ & $216(7.1)$ & 0.729 \\
\hline Leukemia, n (\%) & $37(1.2)$ & $130(1.8)$ & 0.025 & $37(1.2)$ & $39(1.3)$ & 0.817 \\
\hline Malignant lymphoma, n (\%) & $24(0.8)$ & $85(1.2)$ & 0.066 & $24(0.8)$ & $22(0.7)$ & 0.767 \\
\hline Severe liver disease, n (\%) & $34(1.1)$ & $109(1.5)$ & 0.100 & $34(1.1)$ & $30(1.0)$ & 0.615 \\
\hline Metastasis tumor, n (\%) & $137(4.3)$ & $316(4.3)$ & 0.921 & $136(4.5)$ & $142(4.7)$ & 0.713 \\
\hline
\end{tabular}

MDR multi-drug resistant, $C C I$ Chalson comorbidity index, ICU intensive care unit, $P S M$ propensity score matching

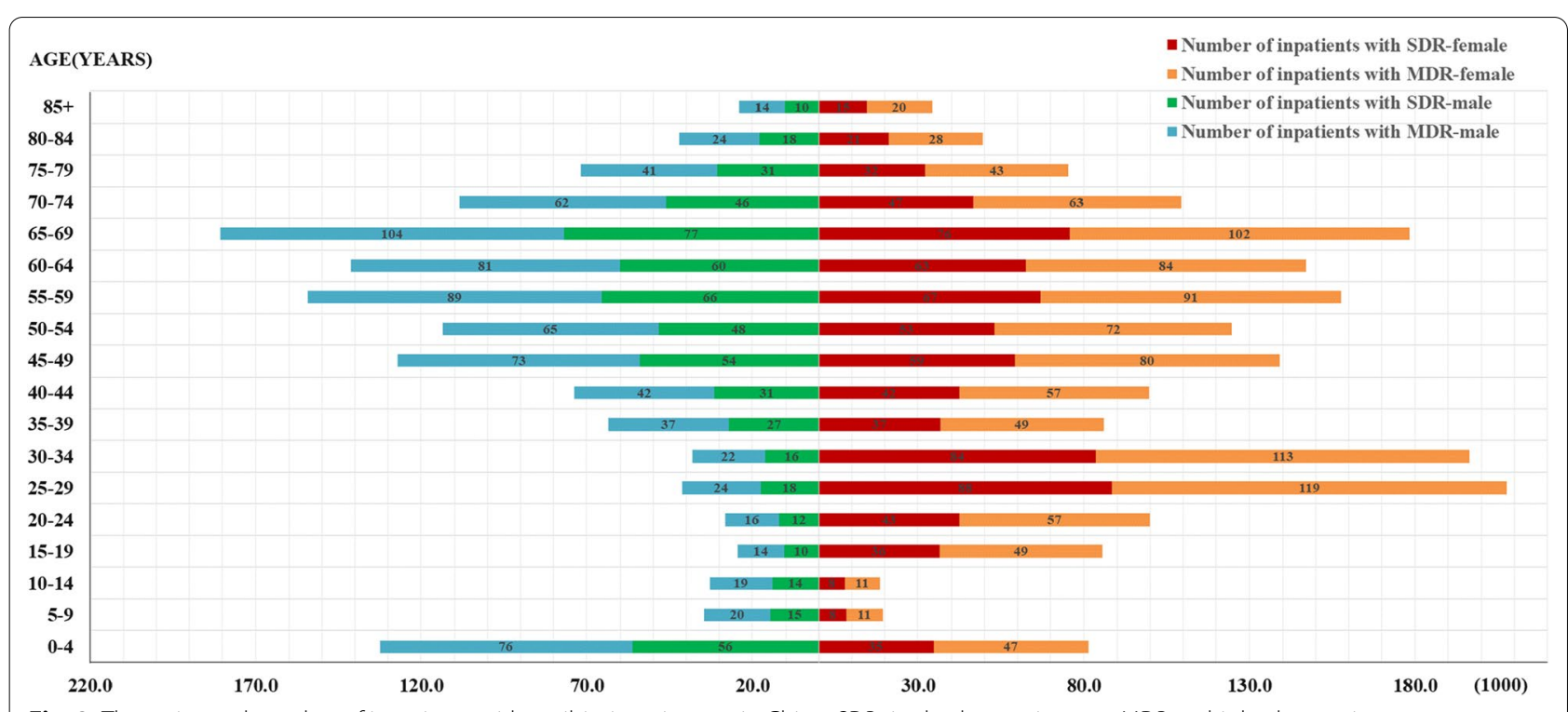

Fig. 2 The estimated number of inpatients with antibiotic resistance in China. SDR single-drug resistance, MDR multiple-drug resistance 
Table 4 Total hospital cost, length of hospital stay, and in-hospital mortality of inpatients with SDR and susceptible infection or colonisation

\begin{tabular}{|c|c|c|c|c|c|c|c|c|c|}
\hline \multirow[t]{2}{*}{ Inpatients } & \multicolumn{3}{|c|}{ Total hospital cost (\$) } & \multicolumn{3}{|c|}{ Length of hospital stay (days) } & \multicolumn{3}{|c|}{ In-hospital mortality rate (\%) } \\
\hline & Mean & $95 \%$ UI & & Mean & $95 \%$ & & Rate & $95 \%$ & \\
\hline Susceptible & 9558 & 9432 & 9684 & 22.01 & 21.72 & 22.29 & 1.92 & 1.80 & 2.04 \\
\hline SDR & 10,702 & 10,576 & 10,827 & 26.07 & 25.81 & 26.34 & 2.67 & 2.53 & 2.82 \\
\hline Difference & 1144 & 965 & 1322 & 4.09 & 3.70 & 4.47 & 0.78 & 0.59 & 0.96 \\
\hline
\end{tabular}

SDR single-drug resistant, UI uncertainty interval

Table 5 Total hospital cost, length of hospital stay, and in-hospital mortality of inpatients with MDR and susceptible infection or colonisation

\begin{tabular}{|c|c|c|c|c|c|c|c|c|c|}
\hline \multirow[t]{2}{*}{ Inpatients } & \multicolumn{3}{|c|}{ Total hospital cost $(\$)$} & \multicolumn{3}{|c|}{ Length of hospital stay (days) } & \multicolumn{3}{|c|}{ In-hospital mortality rate (\%) } \\
\hline & Mean & $95 \%$ UI & & Mean & $95 \% \mathrm{~L}$ & & Rate & $95 \%$ & \\
\hline Susceptible & 9616 & 9492 & 9739 & 22.20 & 21.91 & 22.48 & 2.08 & 1.95 & 2.20 \\
\hline MDR & 13,017 & 12,857 & 13,176 & 27.70 & 27.44 & 27.96 & 3.58 & 3.41 & 3.74 \\
\hline Difference & 3391 & 3188 & 3594 & 5.48 & 5.10 & 5.87 & 1.50 & 1.29 & 1.70 \\
\hline
\end{tabular}

$M D R$ multi-drug resistant, $U /$ uncertainty interval

Table 6 Economic burden caused by inpatients with SDR and MDR infection or colonisation in China

\begin{tabular}{|c|c|c|c|c|c|c|c|c|c|}
\hline \multirow[t]{2}{*}{ Economic burden ( $\$$ billion) } & \multicolumn{3}{|l|}{ SDR } & \multicolumn{3}{|l|}{ MDR } & \multicolumn{3}{|l|}{ ABR } \\
\hline & Mean & \multicolumn{2}{|c|}{$95 \%$ UI } & Mean & \multicolumn{2}{|c|}{$95 \%$ UI } & Mean & \multicolumn{2}{|c|}{$95 \%$ UI } \\
\hline \multicolumn{10}{|l|}{ Direct economic burden } \\
\hline Direct medical cost & 6 & 5 & 7 & 24 & 22 & 25 & 30 & 27 & 32 \\
\hline Direct non-medical cost & 2 & 2 & 2 & 3 & 3 & 4 & 5 & 5 & 6 \\
\hline Direct economic burden & 8 & 7 & 9 & 27 & 25 & 29 & 35 & 32 & 38 \\
\hline \multicolumn{10}{|l|}{ Indirect economic burden } \\
\hline Cost of productivity loss measured in DALYs & 11 & 8 & 13 & 28 & 24 & 32 & 39 & 32 & 45 \\
\hline Cost of care giver & 1 & 1 & 1 & 2 & 2 & 2 & 4 & 3 & 4 \\
\hline Indirect economic burden & 12 & 9 & 15 & 30 & 26 & 34 & 42 & 35 & 49 \\
\hline \multicolumn{10}{|l|}{ Societal economic burden } \\
\hline Socio-economic burden & 20 & 16 & 24 & 57 & 51 & 63 & 77 & 67 & 87 \\
\hline Socio-economic burden accounted for GDP (\%) & 0.10 & 0.08 & 0.11 & 0.27 & 0.25 & 0.30 & 0.37 & 0.32 & 0.42 \\
\hline
\end{tabular}

$S D R$ single-drug resistant, $M D R$ multi-drug resistant, $A B R$ antibiotic resistant, Ul uncertainty interval, $D A L Y_{s}$ disability-adjusted life years, GDP gross domestic product

burden associated with AR in China was $\$ 77$ billion, which caused the similar GDP loss in China and the US despite the diversity in healthcare system settings, suggesting that AMR poses threat to all economies globally and cross-border cooperation is critical to mitigate the negative impact of AMR [10].

Since 2015, a series of national guidelines and recommendations for prudent use of antibiotics was launched to demonstrate the nation's top-level political commitment, including China's National Action Plan of AMR in humans (2016-2020), National Action Plan of AMR in animals (2017-2020), National Administrative Regulations for Clinical Use of Antibacterial Agents, and a national campaign in public hospitals for $\operatorname{AR}[28,29]$. Significant progress has been made in achieving some of the objectives in the National Action Plan of AMR. Legislative enforcement in antibiotic use in paediatric patients and food-producing animals, and national surveillance with timely mandatory reporting and data accessibility highlighted the country's progress, reflected by the reduction in broad-spectrum antibiotic consumptions for surgical prophylaxis and among inpatients; and 
decrease in total hospital resistance and MRSA incidence [30]. Tailored training and research has been embedded in hospitals to guide clinical practice and minimise skill and knowledge gaps in healthcare professionals [30]. However, areas for improvement remain. AMR is absent from the nation's wider public health agenda, including Healthy China 2030, which lacks any strategic measure to address AMR. Shortages of qualified general practitioners and low utilisation of nursing workforce hindered local implementation of top-level policy directive [31, 32]. Organisational regulation relies on administrative power rather than dedicated professional roles. Further, pharmaceutical industry continues incentivising prescription and over-the-counter sales [33]. Low public awareness, driven by limited health literacy about antiinfective and anti-inflammatory drugs, remains a barrier to engaging patients and citizens to promote optimal antimicrobial stewardship and infection prevention [22]. To overcome the challenge, it is essential to establish estimation of the burden of AMR on both public health and economic systems. Our study made contribution in both empirical knowledge and methodology for future research of AMR in China.

Our study has four limitations, which provide the scope for future studies. First, this study is focused on methodology to discuss how to scientifically estimate the economic burden of AR in China, the results may not be the accurate answer to the current economic burden of AR in China. Some variables, such as total hospital cost, length of hospital stay, and in-hospital mortality rate were collected from four tertiary hospitals in this study, and may have limited representativeness when used to predict national level economic burden. To minimise the bias, we validated the data in resistance level using historical statistics on national level. Second, the data is only valid for patients seeking care in the hospital, the impact of AR in the community has not been included. Third, due to the retrospective nature of our study, it is difficult to distinguish between colonisation and infection, which may lead to underestimation of the clinical and economic outcomes of AR infection. AR also impacts patients who do not become infected, and some studies reported that colonisation is associated with increased hospital cost, hospital stay, and hospital mortality [10], therefore, colonisation, as an important reservoir for bacteria causing infection, should be considered as well. Moreover, we only included observed variables in the PSM method, and some hidden bias may remain after matching.

\section{Conclusion}

We estimated a percentage of $27.45 \%$ inpatients with resistant infection or colonisation nationwide in China, of which MDR accounted for $15.77 \%$. we estimated a total societal economic cost attributed to $\mathrm{AR}$ in inpatients in China of $\$ 77$ billion, which is equivalent to $0.37 \%$ of China's GDP in 2017. AR places a significant burden on patient health and healthcare systems. Context-specific interventions are urgently needed to be implemented to promote prudent use of antibiotics in hospitals in China. Quantifying the economic costs of resistant infection or colonisation is the essential step towards building an economic case for global and national actions to combat AMR. It is essential to establish estimation of the burden of AMR on both public health and economic systems. Our study made contribution in both empirical knowledge and methodology for future research of AMR in China.

\section{Supplementary information}

The online version contains supplementary material available at https://doi. org/10.1186/s13756-020-00872-w.

Additional file 1

\section{Abbreviations}

AR: Antibiotic resistance; MRSA: Methicillin-resistant Staphylococcus aureus; VREfm: Vancomycin-resistant Enterococcus faecium; VREfs: Vancomycinresistant Enterococcus faecalis; 3GCREC: Third-generation cephalosporin resistant Escherichia coli; 3GCRKP: Third generation cephalosporin resistant Klebsiella pneumonia; CRKP: Carbapenem resistant Klebsiella pneumonia; CRPA: Carbapenem resistant Pseudomonas aeruginosa; CRAB: Carbapenem resistant Acinetobacter baumannii; GDP: Gross domestic product; US: United States; AMR: Antimicrobial resistance; EMR: Electronic medical records; CCl: Charlson Comorbidity Index; ICU: Intensive care unit; SDR: Single-drug resistant; MDR: Multi-drug resistant; PSM: Propensity score matching; UI: Uncertainty interval; UN: United Nations; DALYs: Disability-adjusted life years.

\section{Acknowledgements}

We want to thank the Center for Health Policy Studies, School of Medicine, Zhejiang University for the assistance in primary data collection.

\section{Authors' contributions}

XZ participated in the conception and design of this study, data collection, data analysis, and interpretation of data, drafted and revised the manuscript. CSL participated in the conception and design of the study and helped in the revising the manuscript. XS and NZ performed the data analysis, and interpretation of data, drafted and revised the manuscript. HD participated in the conception, design of the study, data collection and interpretation of data, and drafted and revised the manuscript. All authors read and approved the final manuscript.

\section{Funding}

This work was supported by "Pfizer Investment Co. Ltd (Burden of multi-drug resistant infections in China and associated risk factors)","The Fundamental Research Funds of Shandong University" and "The joint Research Funds for Shandong University and Karolinska Institutet".

Availability of data and materials

All data analysed during this study are provided in the Tables 1-6 and Figs. 1-2, and Additional file 1.

Ethics approval and consent to participate Not applicable.

Consent for publication

Not applicable. 


\section{Competing interests}

The authors declare that they have no competing interests.

\section{Author details}

${ }^{1}$ Center for Health Policy Studies, School of Public Health, Zhejiang University School of Medicine, Hangzhou, China. ${ }^{2}$ Centre for Health Management and Policy Research, School of Public Health, Cheeloo College of Medicine, (NHC Key Laboratory of Health Economics and Policy Research), Shandong University, Jinan 250012, China. ${ }^{3}$ Department of Global Public Health, Karolinska Institutet, Stockholm, Sweden. ${ }^{4}$ National Institute for Health Research (NIHR) Health Protection Research Unit (HPRU) in Healthcare Associated Infections and Antimicrobial Resistance (HCAls \& AMR), Department of Infectious Disease, Imperial College, London, UK. ${ }^{5}$ Center for Health Policy and Management Studies, Nanjing University, Nanjing, China. ${ }^{6}$ The Fourth Affiliated Hospital Zhejiang University School of Medicine, Yiwu, China.

Received: 6 June 2020 Accepted: 22 December 2020

Published online: 06 January 2021

\section{References}

1. World Health Organization. Antibiotic resistance. 2018. https://www.who. int/news-room/fact-sheets/detail/antibiotic-resistance. Accessed $11 \mathrm{Apr}$ 2020.

2. Heddini A, Cars O, Qiang S, Tomson G. Antibiotic resistance in China-a major future challenge. Lancet. 2009;373:30.

3. Qiao M, Ying GG, Singer AC, Zhu YG. Review of antibiotic resistance in China and its environment. Environ Int. 2018:110:160-72.

4. Wang J, Wang P, Wang X, Zheng Y, Xiao Y. Use and prescription of antibiotics in primary health care settings in China. JAMA Intern Med. 2014;174:1914-20.

5. Van Boeckel TP, Gandra S, Ashok A, Caudron Q, Grenfell BT, Levin SA, et al. Global antibiotic consumption 2000 to 2010: an analysis of national pharmaceutical sales data. Lancet Infect Dis. 2014;14:742-50.

6. Zhang R, Eggleston K, Rotimi V, Zeckhauser RJ. Antibiotic resistance as a global threat: evidence from China, Kuwait and the United States. Global Health. 2006;2:6

7. Centers for Disease Control and Prevention. Antibiotic resistance threats in the United States, 2013. 2013. https://www.cdc.gov/drugresistance/ pdf/ar-threats-2013-508.pdf. Accessed 2018.

8. China Antimicrobial Resistance Surveillance System. Annual report of the China antimicrobial resistance surveillance, 2017. 2018. http://www.carss $\mathrm{cn} /$ Report/Details?ald=552. Accessed 14 Feb 2019.

9. European Center for Disease Prevention and Control. Surveillance of antimicrobial resistance in Europe: annual report of the European Antimicrobial Resistance Surveillance Network (EARS-Net) 2017. 2018. https://ecdc. europa.eu/sites/portal/files/documents/EARS-Net-report-2017-updat e-jan-2019.pdf. Accessed 7 Aug 2019.

10. Zhen $X$, Lundborg CS, Sun X, Hu X, Dong H. Economic burden of antibiotic resistance in ESKAPE organisms: a systematic review. Antimicrob Resist Infect Control. 2019;8:137.

11. European Centers for Disease Control and Prevention. Antimicrobial resistance: trackling the burden in the European Union. 2019. https:// www.oecd.org/health/health-systems/AMR-Tackling-the-Burden-in-theEU-OECD-ECDC-Briefing-Note-2019.pdf. Accessed 4 July 2019.

12. European Centre for Disease Prevention and Control. The bacteria challenge time to react. 2008. https://ecdc.europa.eu/sites/portal/files /media/en/publications/Publications/0909_TER_The_Bacterial_Chall enge_Time_to_React.pdf. Accessed 2018.

13. Centers For Disease Control Prevention. Antibiotic resistance threats in the United States. 2019. https://www.cdc.gov/drugresistance/pdf/threa ts-report/2019-ar-threats-report-508.pdf. Accessed 10 Aug 2020.

14. National Health and Family Plan Commission. National action plan to combat antimicrobial resistance. 2016. http://www.gov.cn/xinwe n/2016-08/25/content 5102348.htm. Accessed 5 Mar 2019.

15. de Kraker ME, Stewardson AJ, Harbarth S. Will 10 million people die a year due to antimicrobial resistance by 2050? PLoS Med. 2016:13:e1002184.
16. World Health Organization. Global action plan on antimicrobial resistance. 2015. https://apps.who.int/iris/bitstream/handle/10665/19373 6/9789241509763_eng.pdf?sequence=1. Accessed 12 Feb 2019.

17. Zhen X, Chen Y, Hu X, Dong P, Gu S, Sheng YY, et al. The difference in medical costs between carbapenem-resistant Acinetobacter baumannii and non-resistant groups: a case study from a hospital in Zhejiang province, China. Eur J Clin Microbiol Infect Dis. 2017:36:1989-94.

18. Magiorakos AP, Srinivasan A, Carey RB, Carmeli Y, Falagas ME, Giske CG, et al. Multidrug-resistant, extensively drug-resistant and pandrugresistant bacteria: an international expert proposal for interim standard definitions for acquired resistance. Clin Microbiol Infect. 2012;18:268-81.

19. Li H, Graham DJ, Majumdar A. The impacts of speed cameras on road accidents: an application of propensity score matching methods. Accid Anal Prev. 2013;60:148-57.

20. Cassini A, Hogberg LD, Plachouras D, Quattrocchi A, Hoxha A, Simonsen GS, et al. Attributable deaths and disability-adjusted life-years caused by infections with antibiotic-resistant bacteria in the EU and the European Economic Area in 2015: a population-level modelling analysis. Lancet Infect Dis. 2019;19:56-66.

21. National Health Commission of the People's Republic of China. 2018 China Health Statistical Yearbook. 2018. http://www.nhc.gov.cn/wjw/tjnj/ list.shtml. Accessed April 162019.

22. United Nations. World population prospects 2019. 2019. https://popul ation.un.org/wpp/Download/Standard/Mortality/. Accessed 18 August 2019.

23. Organization for Economic Cooperation and Development. Purchasing power parities for GDP. 2019. https://stats.oecd.org/index.aspx?query $\mathrm{id}=221 \#$. Accessed 15 Feb 2019.

24. Organization for Economic Cooperation and Development. Consumer price indices. 2019. https://stats.oecd.org/index.aspx?queryid =221\#. Accessed 15 Feb 2019.

25. Zhen X, Zhang H, Hu X, Gu S, Li Y, Gu Y, et al. A comparative study of catastrophic health expenditure in Zhejiang and Qinghai province. China BMC Health Serv Res. 2018;18:844.

26. National Bureau of Statistics. China statistical yearbook 2018. 2018. http:// www.stats.gov.cn/tjsj/ndsj/2018/indexch.htm. Accessed 16 Apr 2019.

27. Chen X. Health economic. Beijing: People's Medical Publishing House 2013.

28. Xiao Y, Zhang J, Zheng B, Zhao L, Li S, Li L. Changes in Chinese policies to promote the rational use of antibiotics. PLoS Med. 2013. https://doi. org/10.1371/journal.pmed.1001556.

29. Xiao Y, Li L. China's national plan to combat antimicrobial resistance. Lancet Infect Dis. 2016;16:1216-8.

30. Zhu N, Castro-Sánchez E, Zhen X, Holmes A, Ferlie E, Ahmad R. Addressing Antimicrobial resistance in China: progress and challenges in translating political commitment into national action. In The 19th international congress of infectious diseases: conference proceedings; 2019.

31. Sun Q, Dyar OJ, Zhao L, Tomson G, Nilsson LE, Grape M, et al. Overuse of antibiotics for the common cold - attitudes and behaviors among doctors in rural areas of Shandong Province. China BMC Pharmacol Toxicol. 2015. https://doi.org/10.1186/s40360-015-0009-x.

32. Wei X, Zhang Z, Walley J, Hicks J, Zeng J, Deng S, et al. Effect of a training and educational intervention for physicians and caregivers on antibiotic prescribing for upper respiratory tract infections in children at primary care facilities in rural China: a cluster-randomised controlled trial. Lancet Global Health. 2017;5:e1258-67.

33. Chang J, Xu S, Zhu S, Li Z, Yu J, Zhang Y, et al. Assessment of non-prescription antibiotic dispensing at community pharmacies in China with simulated clients: a mixed cross-sectional and longitudinal study. Lancet Infect Dis. 2019:12:1345-54.

\section{Publisher's Note}

Springer Nature remains neutral with regard to jurisdictional claims in published maps and institutional affiliations. 